\title{
Stability analysis of a partitioned iterative method for steady free surface flow
}

\author{
Toon Demeester ${ }^{\mathrm{a}, *}$, Joris Degroote $^{\mathrm{a}}$, Jan Vierendeels ${ }^{\mathrm{a}}$ \\ ${ }^{a}$ Department of Flow, Heat and Combustion Mechanics, Ghent University, Sint-Pietersnieuwstraat 41, B-9000 Ghent, Belgium
}

Keywords: Free surface flow, Fitting method, Modal analysis, Dynamic boundary condition, Gauss-Seidel

\section{Introduction}

This note considers the steady free surface (FS) flow problem as encountered in the paper by van Brummelen et al. [1]. In that paper, steady flow of water in a two-dimensional slice of an infinitely wide open channel with a particular bottom wall is calculated as the first step in the development of a 3D surface fitting method for steady flow around ships. In these water-air flows, the influence of air is usually negligible due to the large difference in density. Contrary to surface capturing methods which are typically multiphase techniques (such as the volume-of-fluid method), fitting methods usually consider only the water phase. The latter approach requires appropriate FS boundary conditions. The dynamic boundary condition (DBC) used here assumes that the pressure is constant (atmospheric) at the FS and the shear stresses are zero. The kinematic boundary condition (KBC) states that the FS is impermeable.

Fitting methods typically consist of an iterative process with two steps that are repeated until convergence is reached in each time step: first calculation of the flow field with a fixed FS position and suitable boundary conditions, and then update of the FS position. Often the DBC is used in the first step, the KBC in the second [2,3]. Using the $\mathrm{KBC}$ for updating the surface results in a time-stepping method, which is not efficient for steady cases due to the large number of time steps before transient phenomena have disappeared. Van Brummelen [1] describes a steady iterative fitting method which uses the DBC for the surface update and a combined boundary condition (KBC + DBC) in the flow solver. This method is efficient, but because the pressure gradient and the vertical velocity are both calculated implicitly at the FS [4], it requires access to the source code of a coupled solver, making it less flexible. Combining a steady iterative approach with a black box solver requires that the boundary conditions in the flow solver are easy to implement (only $\mathrm{KBC}$ ) and that the update is time-independent (DBC).

A modal analysis is carried out in this note to investigate the relation between position and pressure perturbation errors at the FS for the latter boundary conditions. A von Neumann stability analysis of Gauss-Seidel iterations is then performed, which iterate between solving the flow and updating the surface.

\section{Modal analysis}

The case studied in the modal analysis is inviscid FS flow over a flat bottom, which has a horizontal surface as solution. A small perturbation $\tilde{\eta}$ of the FS height is considered, which can be split into its Fourier-modes $a_{0}, a_{i} \sin i k x$ and $b_{i} \cos i k x$ with $i \in \mathbb{N}_{0}$ and $k=2 \pi / \lambda$ the wavenumber. Sine and cosine modes will lead to the same flow field shifted over $\lambda / 4$, so only sines are studied. The constant mode $a_{0}$ is not of interest: it leads to a constant pressure at the FS, and is therefore also a solution of the FS flow problem. The problem studied is shown in Fig. 1: a periodic flow with FS position error

$$
\tilde{\eta}(x)=a \sin k x
$$

\footnotetext{
${ }^{*}$ Corresponding author

Email addresses: toon.demeester@ugent.be (Toon Demeester), joris.degroote@ugent.be (Joris Degroote), jan.vierendeels@ugent.be (Jan Vierendeels)
} 


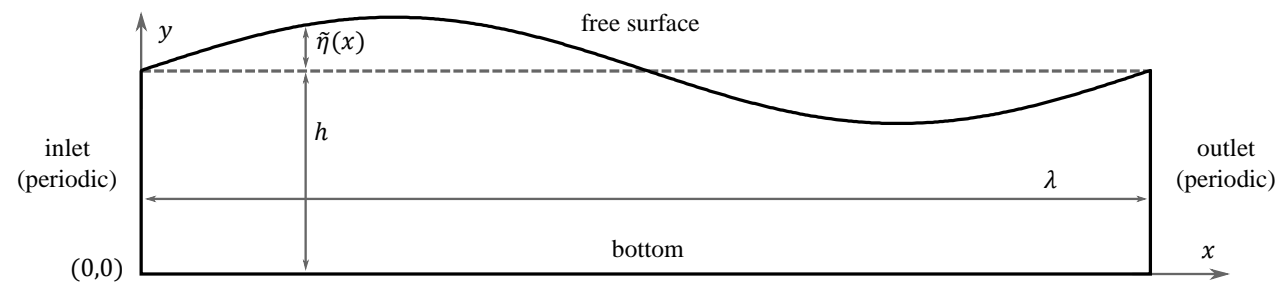

Figure 1: Domain studied in modal analysis

and average inflow-velocity $U$. The bottom and FS are considered impermeable free-slip walls.

The modal analysis is performed in two ways. A potential flow solution is derived analytically, relating the FS pressure error $\tilde{p}$ to the FS position error $\tilde{\eta}$. The results are then checked against numerical simulations using a finite volume flow solver.

\subsection{Potential flow solution}

The flow field is derived for a small perturbation $\tilde{\eta}$, resulting in two conditions on the error amplitude:

$$
a \ll \lambda \quad \text { and } \quad a \ll h
$$

The complex potential $w$ of this flow is

$$
w=\frac{a U}{\sinh k h} \cos (k z)+U z=\phi+i \psi \quad \text { with } \quad z=x+i y
$$

The velocity potential $\phi$ and stream function $\psi$ are defined as

$$
\boldsymbol{u}=[u, v]=\left[\frac{\partial \phi}{\partial x}, \frac{\partial \phi}{\partial y}\right]=\left[\frac{\partial \psi}{\partial y},-\frac{\partial \psi}{\partial x}\right]
$$

with $\boldsymbol{u}$ the velocity vector. It can be proven that $w$ gives the correct flow field by verifying that the velocity is indeed periodic between inlet and outlet and that $\psi$ is constant along the bottom and the free surface. The former is straightforward to prove, the latter is done in [5] for a similar flow field.

The velocity $\boldsymbol{u}$ is found from (4), the pressure $p$ is derived from the Bernoulli equation which reduces to

$$
p=-\frac{\rho \boldsymbol{u}^{2}}{2}-\rho g y+C=-\frac{\rho U^{2}}{2}\left[1+\left(\frac{a k}{\sinh k h}\right)^{2}\left(\sinh ^{2} k y+\sin ^{2} k x\right)-2 \frac{a k}{\sinh k h} \cosh k y \sin k x\right]-\rho g y+C
$$

The FS pressure error $\tilde{p}$ is found by substituting $y=h+\tilde{\eta}$ and choosing the constant $C$ so that $p$ is zero at the intersection between the FS and the inlet (coordinate $[0, h]$ ), giving

$$
\begin{aligned}
\tilde{p} & =-\frac{\rho U^{2}}{2}\left[1+\left(\frac{a k}{\sinh k h}\right)^{2}\left(\left(1+O\left(\frac{a}{\lambda}\right)\right) \sinh ^{2} k h+\sin ^{2} k x\right)-2 \frac{a k}{\sinh k h}\left(1+O\left(\frac{a}{\lambda}\right)\right) \cosh k h \sin k x\right]-\rho g y+C \\
& \approx-\frac{\rho U^{2}}{2}\left[1+(a k)^{2}+\left(\frac{a k \sin k x}{\sinh k h}\right)^{2}-2 \frac{a k \sin k x}{\tanh k h}\right]-\rho g(h+a \sin k x)+\frac{\rho U^{2}}{2}\left(1+(a k)^{2}\right)+\rho g h \\
& =-\frac{\rho U^{2} k a \sin k x}{\tanh k h}\left[\frac{a k \sin k x \tanh k h}{2 \sinh ^{2} k h}-1\right]-\rho g a \sin k x=-\frac{\rho U^{2} k a \sin k x}{\tanh k h}\left[O\left(\frac{a}{h}\right)-1\right]-\rho g a \sin k x \\
& \approx \rho g a \sin k x\left[\frac{U^{2}}{g h} \frac{k h}{\tanh k h}-1\right]=\rho g\left[\operatorname{Fr}^{2} \frac{k h}{\tanh k h}-1\right] \tilde{\eta}
\end{aligned}
$$

In (6) a formal expansion is used to approximate the terms containing $y$. The terms of $O(a / \lambda)$ in (6) and the one of $O(a / h)$ in (8) are discarded following the conditions in (2). The Froude number $\mathrm{Fr}=U / \sqrt{g h}$ is introduced in the last 
equality. It follows that the FS pressure error $\tilde{p}$ is directly proportional to the position error $\tilde{\eta}$, leading to the definition of the proportional factor $K_{\mathrm{th}}$ as

$$
K_{\mathrm{th}}=\frac{1}{\rho g\left[\mathrm{Fr}^{2} \frac{k h}{\tanh k h}-1\right]} \quad \text { with } \quad \tilde{\eta}=K_{\mathrm{th}} \cdot \tilde{p}
$$

This result is valid for both sine and cosine modes. Two interesting cases can be distinguished. For quiescent water, i.e. $\operatorname{Fr}=0$, the relation between $\tilde{\eta}$ and $\tilde{p}$ reduces to the hydrostatic equation. For shallow water expression (10) reduces to

$$
K_{\mathrm{th}} \approx \frac{1}{\rho g\left[\mathrm{Fr}^{2}-1\right]} \quad(k h=2 \pi h / \lambda \ll 1)
$$

which can be derived directly from the Bernoulli equation on a streamline, assuming that the velocity is uniform over the depth and linearizing the result.

\subsection{Flow simulations}

The result in (10) was checked numerically with a finite volume flow solver for a large range of the two relevant dimensionless parameters which appear in $K_{\mathrm{th}}$ : Fr and $h / \lambda=k h / 2 \pi$. The Froude number describes the relative importance of inertial to gravitational forces. The ratio $h / \lambda$ is the aspect ratio of the domain and measures how deep or shallow the flow is relative to the wavelength. The examined ranges are $\operatorname{Fr} \in[0.2,2.0]$ and $h / \lambda \in[1 / 8,8]$.

The inlet and outlet boundary conditions should ideally be periodic, but this is impractical for inviscid flow simulations, as there is no physical pressure drop over the domain. Instead, the velocity was imposed at the inlet by taking the analytically derived values resulting from the potential analysis. Gravity is not considered directly, so that the hydrodynamic pressure $\varphi=p+\rho g y$ is calculated in the flow solver and the static pressure $p$ is obtained by post-processing. Variations in $\varphi$ at the outlet are much smaller than at the FS, more precisely

$$
\frac{\left.\Delta \varphi\right|_{\text {outlet }}}{\left.\Delta \varphi\right|_{\mathrm{FS}}}=\frac{\pi}{2} \tanh (k h) \frac{a}{\lambda} \ll 1 \quad \text { with } \quad \Delta \varphi=\max (\varphi)-\min (\varphi)
$$

so that the outlet pressure $\varphi$ in the simulations can be assumed constant to good approximation.

The domain was discretized using a structured mesh of $600 \times 600$ cells. The amplitude $a$ was defined as a function of $\lambda$ and $h$ as

$$
a=f \min (\lambda, h)
$$

with $f=1 / 200$ so that $\tilde{\eta}$ could be regarded as a small perturbation. It is shown later that the solutions are independent of both the mesh size and the error amplitude.

\section{Comparison of analytical and numerical results}

The analytical result $K_{\text {th }}$ from (10) is plotted in Fig. 2 as a function of Fr and $h / \lambda$. An asymptote splits the domain in a region where gravitational forces dominate the flow (lower left corner) and a region where inertial forces dominate. Close to the asymptote, the opposing effects of gravity and inertia on $\tilde{p}$ balance each other out. Along the asymptote the phase velocity of the waves is equal to the flow velocity, giving rise to steady surface gravity waves. These are correct solutions of the linearized free surface problem $(\tilde{p}=0, \mathrm{DBC}$ satisfied $)$ and therefore give $K_{t h}=\infty$.

The finite volume simulations described in Section 2.2 lead to a pressure error $\tilde{p}$ for given surface error $\tilde{\eta}$. According to the potential flow solution, there should be a proportional relation between the two. To check this, the proportional part $\tilde{p}_{1, \sin }=a_{1, \sin } \sin k x$ is extracted from $\tilde{p}$ using the Discrete Fourier Transform. Similar to $K_{\text {th }}$ in (10), a proportional factor $K_{\text {num }}$ can be defined for the simulations as

$$
K_{\text {num }}=\frac{a}{a_{1, \text { sin }}} \quad \text { with } \quad \tilde{\eta}=K_{\text {num }} \cdot \tilde{p}_{1, \text { sin }}
$$




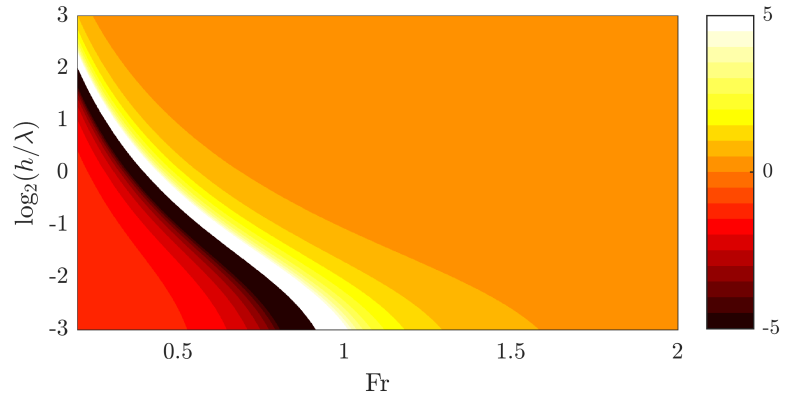

Figure 2: The analytical proportional factor $\rho g K_{\text {th }}$

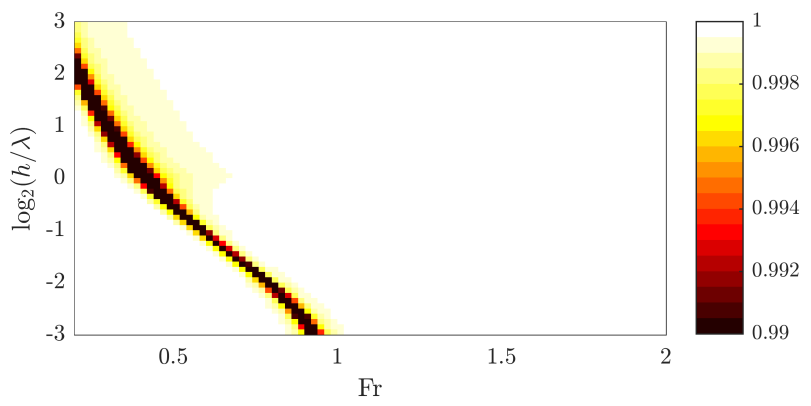

Figure 4: Importance of proportional contribution $R$

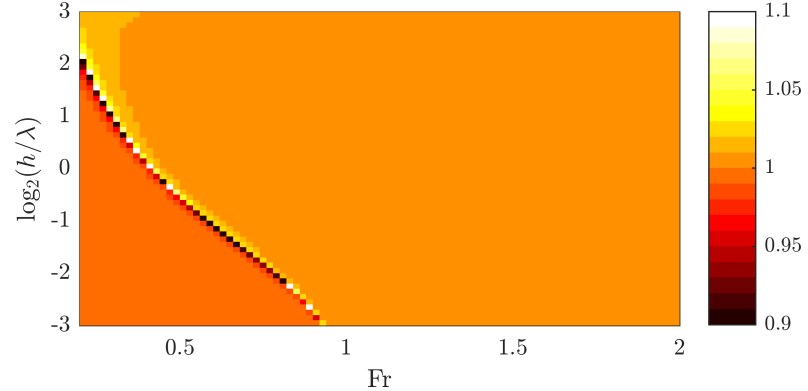

Figure 3: Ratio of numerical and analytical factor $K_{\text {num }} / K_{\text {th }}$

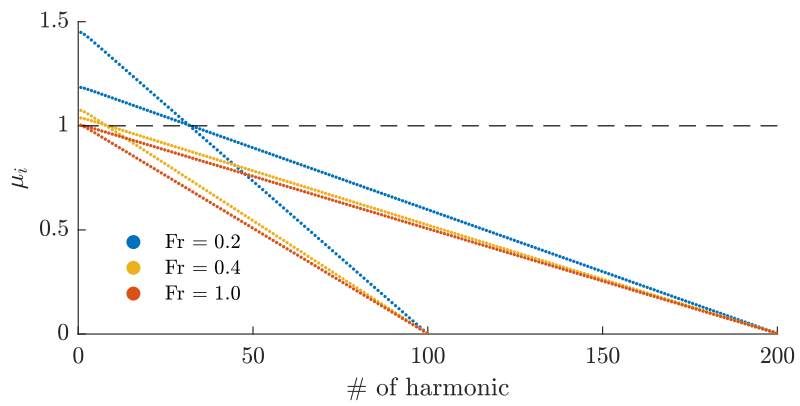

Figure 5: Amplification $\mu$ in Gauss-Seidel iterations for 100 and 200 harmonics

The ratio of $K_{\text {th }}$ and $K_{\text {num }}$ is shown in Fig. 3. In most of the domain $K_{\text {num }} / K_{\text {th }}$ lies between 0.99 and 1.01 ; only close to the asymptote there are significant differences because $K_{\text {th }}$ changes very fast in this region.

The importance of the proportional component $\tilde{p}_{1 \text {,sin }}$ in $\tilde{p}$ can be seen by comparing their rms-values through the ratio

$$
R=\frac{\operatorname{rms}\left(\tilde{p}_{1, \sin }\right)}{\operatorname{rms}(\tilde{p})} \quad \text { with } \quad 0 \leq R \leq 1
$$

where the numerator is found as $\left|a_{1, \sin }\right| / \sqrt{2}$. If $R<1, \tilde{p}$ contains higher harmonics and/or a phase shift of the base harmonic. $R$ is plotted in Fig. 4 : the deviation is smaller than $0.1 \%$, except next to the asymptote. Here, the pressure error is practically zero in the simulations and its waveform is strongly deformed.

Mesh independence was checked by comparing the values of $K_{\text {num }}$ on the $600 \times 600$ mesh, with those on a $300 \times 300$ mesh. The relative difference between them had an average of $1.2 \%$ and a median of $0.5 \%$ for the same cases as plotted in Fig. 3. The same was done for the perturbation amplitude $a$ : $K_{\text {num }}$ was compared for $f=1 / 200$ and $f=1 / 300$, with $f$ defined in (13). The relative difference between them had an average of $0.12 \%$ and a median of $0.07 \%$.

\section{Stability of Gauss-Seidel iterations}

The stability of Gauss-Seidel iterations is studied for a case similar to that in Fig. 1, but with multiple modes superimposed (i.e. different $h / \lambda$ ), forming an arbitrary perturbation of the FS. The iterative procedure consists of alternating the flow solver $\mathcal{F}$ and the surface update $\mathcal{S}$, forming the system

$$
\left\{\begin{array}{l}
p=\mathcal{F}(\eta) \\
\eta \quad \mathcal{S}(p) \quad \text { with } \quad \mathcal{S}(p)=\eta-K^{*} \cdot \tilde{p}
\end{array}\right.
$$

where $K^{*}$ is calculated as in (10) for a selected wavenumber $k^{*}$ as explained further. The residual operator $\mathcal{R}(\eta)$ is then

$$
\mathcal{R}(\eta)=\mathcal{S}(\mathcal{F}(\eta))-\eta=-K^{*} \tilde{p}
$$


so that the Gauss-Seidel update of iteration $n$ (without underrelaxation) is

$$
\eta^{n+1}=\eta^{n}+\mathcal{R}^{n}=\eta^{n}-K^{*} \tilde{p}^{n}
$$

The errors can be found by subtracting the correct FS from both sides. The amplification $\mu_{i}$ of a mode $\tilde{\eta}_{i}=a_{i} \sin i k x$ is

$$
\mu_{i}=\frac{\tilde{\eta}_{i}^{n+1}}{\tilde{\eta}_{i}^{n}}=1-K^{*} \frac{\tilde{p}_{i}^{n}}{\tilde{\eta}_{i}^{n}}=1-\frac{K^{*}}{K_{\mathrm{th}, i}}
$$

where relation (10) is used in the last step to model the flow solver. Only if $\left|\mu_{i}\right|<1$, error mode $i$ will damp out during Gauss-Seidel iterations and is stable. If modes are present in the left region of Fig. 2 (low Fr), it is not possible to choose a $K^{*}$ for which all modes are stable, because $K_{\mathrm{th}, i}$ changes sign when the asymptote is crossed. However it is possible to make all high wavenumber modes stable by choosing $k^{*}=k_{\max }=\pi / \Delta x$ (with $\Delta x$ the mesh width), leading to a limited number of unstable low wavenumber modes. As an example, $\mu_{i}$ is plotted in Fig. 5 for a mesh with 100 and one with 200 harmonics. The base mode corresponds to $h / \lambda=1 / 8$, so that quite some unstable modes are produced for low Fr. If the base harmonic has a higher $h / \lambda$ or the Froude number is higher, the number of unstable modes reduces quickly. In the fluid-structure interaction community, similar iterative processes with a limited number of unstable modes are often stabilized using a quasi-Newton (QN) coupling algorithm such as IQN-ILS [6]. QN algorithms can also accelerate convergence of badly damped modes ( $\mu$ close to 1). However, when the mesh is refined, $\Delta x \rightarrow 0$ and hence $K^{*}$ becomes proportional to $\Delta x$. As a consequence the number of badly damped modes increases approximately as $1 / \Delta x$, e.g. compare $\mu$ for 100 and 200 harmonics in Fig. 5. The number of required QN coupling iterations increases with the same rate, which means the currently proposed method has scaling problems. The steady gravity wave has $\mu=1$ and therefore no damping. This mode may be present in the solution, but its magnitude and phase are determined by the boundary conditions. Future numerical tests will show if additional treatment for this mode is necessary.

It must be noted that the theoretical prediction of $\mu_{i}$ in (19) will not be accurate for the highest wavenumber modes on a given mesh, as these consist of only a couple of cells and will therefore give numerical deviations. Furthermore, if the amplitude $a$ is such that the error cannot be considered a perturbation anymore, the flow field may also be different than predicted and lead to deviant values for $K_{\text {num }}$ or even the presence of higher harmonics in $\tilde{p}$. Both effects require further investigation, and a different choice of $K^{*}$ may then be advantageous.

\section{Conclusion}

The steady iterative free surface fitting method described by van Brummelen et al. [1] needs a dedicated flow solver due to the boundary condition at the free surface. This note investigates whether there are alternatives by analyzing free surface flow in a channel with flat bottom. First a relation was derived between the pressure and position error at the free surface. Results from potential flow theory and numerical simulations are in very good agreement. Secondly a stability analysis was done to check the convergence of Gauss-Seidel iterations using the previously derived relation. Depending on the flow conditions, a limited number of low wavenumber modes is expected to be slightly unstable during Gauss-Seidel iterations. Convergence of unstable and badly damped modes may be improved with a quasiNewton algorithm, although the currently proposed method shows scaling problems in that case. Still, the analysis made in this note opens perspectives for new surface fitting techniques.

\section{References}

[1] E. H. Van Brummelen, H. C. Raven, B. Koren, Efficient numerical solution of steady free-surface Navier-Stokes flow, Journal of Computational Physics 174 (1) (2001) 120-137.

[2] G. Tzabiras, A numerical investigation of 2D, steady free surface flows, International Journal for Numerical Methods in Fluids 25 (5) (1997) 567-598.

[3] S. Muzaferija, M. Perić, Computation of free-surface flows using the finite-volume method and moving grids, Numerical Heat Transfer 32 (4) (1997) 369-384.

[4] J. Wackers, B. Koren, H. Raven, A. Van der Ploeg, A. Starke, G. Deng, P. Queutey, M. Visonneau, T. Hino, K. Ohashi, Free-surface viscous flow solution methods for ship hydrodynamics, Archives of Computational Methods in Engineering 18 (1) (2011) 1-41.

[5] L. M. Milne-Thomson, Theoretical hydrodynamics, Courier Corporation, 1968.

[6] J. Degroote, K.-J. Bathe, J. Vierendeels, Performance of a new partitioned procedure versus a monolithic procedure in fluid-structure interaction, Computers \& Structures 87 (11) (2009) 793-801. 